\title{
Product Update
}

\section{ADVISORIES}

Takeda Canada Inc. and Health Canada inform consumers and health care professionals that the product monograph for Actos (pioglitazone) has been updated with new safety information regarding a potential risk of bladder cancer in patients using pioglitazone to manage their diabetes. In a 5-year interim analysis of an ongoing 10-year observational study in patients with diabetes, pioglitazone was associated with an increased risk (HR 1.4, 95\% CI 0.9-2.1) of bladder cancer when taken longer than 12 months compared to patients who were not exposed to the drug. The risk, however, reached statistical significance when pioglitazone was taken longer than 24 months (HR 1.4, 95\% CI 1.03-2.0). Therefore, there may be a small increased risk of bladder cancer in patients treated with pioglitazone for longer durations and high cumulative doses. Further, a possible risk with short-term treatment of pioglitazone cannot be excluded at this time. Actos is contraindicated in patients with active bladder cancer, a history of bladder cancer or uninvestigated macroscopic hematuria. Prior to initiation of treatment with Actos, patients should be assessed for risk factors for bladder cancer that include a family history of bladder cancer, radiation therapy and smoking. Advise patients taking Actos to seek immediate medical attention if they see blood in their urine, present with an increased need to urinate or experience pain while urinating.

After a review of postmarketing reports, GlaxoSmithKline Inc. and Health Canada inform consumers and health care professionals that the product monograph for
Benlysta (belimumab) has been updated with new safety information pertaining to serious hypersensitivity and infusion reactions that can be fatal. Benlysta is indicated, in addition to standard therapy, for reducing disease activity in adult patients with active, autoantibody-positive, systemic lupus erythematosus. Patients with a history of multiple drug allergies or significant hypersensitivity may be at a higher risk for these reactions. It may be beneficial to review and update the patients' list of allergies, especially to drugs and injections, prior to administration. Monitor for infusion and hypersensitivity reactions on the day of the treatment and also after, as these reactions can be delayed. Advise patients to seek medical attention immediately if they develop symptoms of hypersensitivity reactions such as difficulty breathing, hives, lightheadedness, slow heart rate, shortness of breath or swelling of face, mouth, lips or tongue after treatment with Benlysta.

Health Canada informs consumers and health care professionals of changes to the product monograph for Cipralex (escitalopram), which include a warning on dose-dependent prolongations of the QT interval and revised dosing recommendations. Cipralex is contraindicated in patients with congenital long QT syndrome or known QT interval prolongation. According to the product monograph, postmarketing cases of QT interval prolongation and ventricular arrhythmia including torsade de pointes have been reported predominantly in female patients, those with hypokalemia, or those with pre-existing QT interval prolongation or other cardiac diseases. The co-administration of Cipralex is discouraged in patients who are on class $1 \mathrm{~A}$ and III antiarrhythmics, an- tipsychotics such as ziprasidone, tricyclic antidepressants, opioids such as methadone, antimicrobials such as moxifloxacin and medications that can disrupt electrolyte levels. The maximum daily dose is 10 $\mathrm{mg}$ for patients with hepatic impairment, patients who are $\geq 65$ years or those who take cimetidine or omeprazole concomitantly. For all others, the recommended maximum daily dose is $20 \mathrm{mg}$. Advise patients to discuss further with their health care providers, especially before stopping or reducing the dose of Cipralex.

Vita Health Products is conducting a voluntary recall of 5 lots of Compliments Extra Strength Acetaminophen Gelcaps $500 \mathrm{mg}$ (bottles of 120 gelcaps) from the Canadian market as a result of a labelling error. According to the information on the bottles, the product's packaging is child-resistant. The product, however, has been packaged with a non-child-resistant cap. This poses a potential risk to children who may be able to open these bottles and ingest their contents, which could result in serious health consequences. The lots affected are 1B01829H9, 1B01929H9, 1E1742EGG, 1I10102GMB and 1K1672KME. Vita Health Products is requesting that retailers immediately stop the sale of the affected products and return these products to the company. Consumers are advised to contact Vita Health Products at 1-800-665-8820 should they require more information and report any actual and potential adverse reactions to Health Canada.

Celgene Inc. and Health Canada inform consumers and health care professionals that the product monograph of Revlimid (lenalidomide) has been updated 


\section{ADVISORIES (cont'd)}

with new safety information regarding the increased risk of secondary primary malignancies (SPM) in previously treated multiple myeloma patients receiving lenalidomide and dexamethasone (3.98 per 100 patient-years) compared to controls (1.38 per 100 patient-years). SPM include noninvasive cancers such as basal cell or squamous cell skin cancers and invasive cancers, such as solid tumor malignancies. In clinical trials, a 4-fold increased incidence in SPM was observed in patients with newly diagnosed multiple myeloma receiving Revlimid (not an approved Health Canada indication). Only prescribers and pharmacists registered with RevAid, a controlled distribution program, are able to prescribe and dispense Revlimid. Advise patients to talk to their health care providers regarding concerns about any increased risk of having other cancers prior to or during treatment with Revlimid.

Amgen Canada Inc. and Health Canada inform consumers and health care professionals that the product monograph of Xgeva (denosumab) has been updated with new safety information regarding severe symptomatic hypocalcemia (estimated rate $1 \%-2 \%$ from postmarketing cases), which may be fatal. This human monoclonal antibody is indicated in patients with bone metastases from breast cancer, prostate cancer, non-small-cell lung cancer and other solid tumors to reduce the risk of developing skeletal-related events. Patients with severe renal impairment $(\mathrm{CrCl}<30 \mathrm{~mL} / \mathrm{min})$ or those receiving dialysis and not receiving calcium supplementation may be at higher risk of severe symptomatic hypocalcemia. Severe hypocalcemia, defined as corrected serum calcium $<1.75 \mathrm{mmol} / \mathrm{L}$, was reported in $3.1 \%$ of patients receiving Xgeva in clinical trials. Serum calcium levels should be monitored and corrected prior to and during treatment with Xgeva, as required. If the patient is taking other medications that can also lower calcium levels, serum calcium levels may need to be monitored more frequently. Calcium, magnesium and vitamin D may be administered, as necessary. Advise patients to seek medical attention if they experience confusion, muscle spasms, twitches or cramps, seizures or abnormal heart rhythm, as these symptoms can be life-threatening.

\section{NEW PRODUCTS}

Fampyra (fampridine $10 \mathrm{mg}$ sustainedrelease tablets by Biogen Idec Canada Inc.) is a potassium channel blocker and the first Health Canada-approved treatment for the symptomatic improvement of walking in adult patients with MS with walking disability (expanded disability status scale 3.5-7). Fampyra can be used with diseasemodifying therapies used in MS. Advise patients that they should see an improvement within the first 4 weeks; if there is no benefit seen within 4 weeks, treatment should be stopped. Contraindications to Fampyra include renal impairment $(\leq 80$

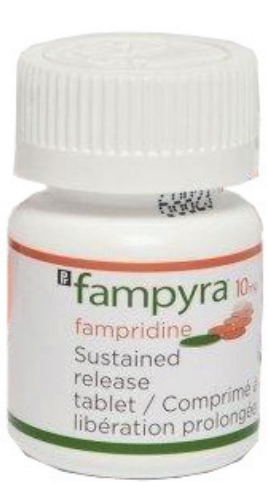
$\mathrm{mL} / \mathrm{min}$ ), prior history or current presentation of seizures and concomitant use of cimetidine and quinidine. The maximum daily dose is $10 \mathrm{mg}$ twice daily, taken 12 hours apart, swallowed whole, with a glass of water and without

food. Taking the doses closer together, taking higher than the recommended maximum daily dose or breaking the sustained-release tablets may increase the risk of seizures. Renal impairment, drug-drug interactions and factors that lower the seizure threshold can also increase the risk of seizures. Evaluate any potential and actual drug-drug interactions prior to initiating Fampyra. For a missed dose, advise patients to take the next dose at the regularly scheduled time and to not take an extra dose. Caution patients about dizziness and balance disorder, especially in the first 4 to 8 weeks of treatment, as this may increase the risk of falls. Other side effects include urinary tract infections, insomnia, headache and nausea.

NYDA (dimeticone 100 cSt Solution 50\% w/w by G. Pohl-Boskamp GmbH \& Co. $\mathrm{KG}$ ) is a topical non-insecticide treatment of scalp hair in case of infestations with head lice (pediculosis). If used as instructed, NYDA is also effective against nymphs and eggs (nits). The silicone-based oil works by flowing into the breathing system of lice, nymphs and embryos in the eggs and blocking the oxygen supply of the central nervous system, thereby resulting in suffocation. NYDA is used in adults and children $\geq 2$ years. As the mixture in NYDA is volatile, instruct patients or caregivers to keep the bottle away from heat and open flames and not to smoke while using the product. Instruct patients or caregivers to flush the eyes with water for 10 to 15 minutes should they come in contact with NYDA.

Samsca (tolvaptan $15 \mathrm{mg}, 30 \mathrm{mg}$ and $60 \mathrm{mg}$ tablets by Otsuka Canada Pharmaceutical Inc.) is a vasopressin $\mathrm{V}_{2}$-receptor antagonist for the treatment of clinically important, non-hypovolemic hyponatremia, e.g., serum sodium $<130 \mathrm{mEq} / \mathrm{L}$ or symptomatic hyponatremia. Samsca is not indicated for patients requiring urgent intervention to raise serum sodium to treat serious neurological symptoms associated with hyponatremia. Contraindications include hypovolemic hyponatremia, urgent need to raise serum sodium acutely, inability of the patient to sense or appropriately respond to thirst, anuric patients and concomitant use of strong CYP3A inhibitors such as ketoconazole, clarithromycin and ritonavir. Instruct patients to avoid concomitant grapefruit juice or grapefruit. The recommended starting dose is $15 \mathrm{mg}$ once daily with or without meals. The daily dose can be increased to $30 \mathrm{mg}$ after at least 24 hours. During initiation and titration of the dose, serum electrolytes and volume should be frequently monitored. Advise patients that they can continue fluid intake in response to thirst while on Samsca. 
Saphris (asenapine $5 \mathrm{mg}$ and $10 \mathrm{mg}$ sublingual tablets by Merck Canada Inc.) is an atypical antipsychotic for the treatment of schizophrenia and for the acute treatment of manic or mixed episodes associated with bipolar I disorder. Saphris can be used as acute monotherapy or co-therapy with lithium or divalproex sodium. Saphris is not indicated in elderly patients with dementia. Instruct patient to place the tablet under the tongue and allow it to dissolve completely (usually within seconds). Saphris undergoes significant first pass effect if swallowed and as such may not be effective. Advise patient not to eat or drink for 10 minutes after the administration to allow for sublingual absorption. The recommended starting or target dose for schizophrenia is $5 \mathrm{mg}$ twice daily. For patients with bipolar I disorder, the dose is $5 \mathrm{mg}$ or $10 \mathrm{mg}$ twice daily. Doses greater than 10 mg twice daily have not been studied for safety and therefore are not recommended. Saphris is not recommended in patients with severe hepatic impairment. There is no dose adjustment required in patients with renal impairment or mild to moderate hepatic impairment. Common side effects include dizziness, fatigue, increased appetite and sleepiness. Advise patients that they may feel a transient sensation of numbness in the tongue or mouth after using Saphris.

Toviaz (fesoterodine fumarate $4 \mathrm{mg}$ and $8 \mathrm{mg}$ extended-release tablets by Pfizer Canada Inc.) is a prodrug and a competitive muscarinic receptor antagonist for the treatment of patients with overactive bladder with symptoms of urinary frequency, urgency or urge incontinence, or any combination of these symptoms. Contraindications include hypersensitivity to tolterodine, uncontrolled narrow-angle glaucoma, gastric retention and urinary retention. The recommended starting dose is $4 \mathrm{mg}$ once daily, swallowed whole with or without food. Toviaz can be taken any time during the day as long as the timing is consistent. The maximum daily dose is 8 mg. For patients with severe renal impairment $(\mathrm{CrCl}<30 \mathrm{~mL} / \mathrm{min})$ and those who are on potent CYP3A4 inhibitors such as clarithromycin, the maximum daily dose is $4 \mathrm{mg}$. Common side effects include dry mouth and constipation. Techniques usually used to help minimize dry mouth, depending on the patient's circumstances, include sipping a little bit of water throughout the day, sucking on ice chips, using an OTC saliva substitute or oral lubricant and avoiding carbonated, caffeinated and alcoholic beverages, salty or spicy foods, as well as mouth rinses that contain alcohol.

\section{Xalkori (crizo-} tinib $200 \mathrm{mg}$ and $250 \mathrm{mg}$ capsules by Pfizer Canada Inc.) is an oral anaplastic lymphoma kinase (ALK)-tyrosine kinase inhibitor for use

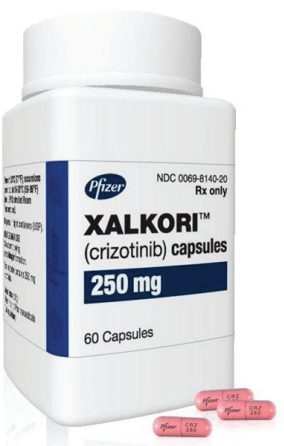
as monotherapy in patients with ALK-positive advanced (not amenable to curative therapy) or metastatic non-small cell lung cancer. Xalkori has been issued marketing authorization with conditions by Health Canada, pending the results of studies to verify its clinical benefit. Contraindications include patients with congenital long QT syndrome or with a persistent Fridericia-corrected electrocardiogram interval of $\geq 500 \mathrm{msec}$. Avoid the concurrent use of strong CYP3A inhibitors and inducers. The recommended oral dose is $250 \mathrm{mg}$ twice daily without regard to meals. Refer to the product monograph for dose modification protocols based on individual safety and tolerability of Xalkori.

Zelboraf (vemurafenib $240 \mathrm{mg}$ film-coated tablet by Hoffmann-La Roche Limited) is an oral inhibitor of the activated form of the BRAF serine-threonine kinase enzyme for the treatment of BRAF V600 mutationpositive unresectable or metastatic melanoma. A validated test is required to identify BRAF V600 mutation status. The recommended dose is $960 \mathrm{mg}$ twice daily. It is recommended that treatment with Zelboraf be continued until disease progression or the development of unacceptable toxicity. Please consult the product monograph for further information on dose adjustments and management of QTc interval prolongation while on Zelboraf.

\section{NEW INDICATIONS}

Prevnar 13 (pneumococcal 13-valent conjugate vaccine by Pfizer Canada Inc.) is now approved for active immunization for the prevention of invasive pneumococcal disease (including sepsis, meningitis, bacteremic pneumonia, pleural empyema and bacteremia) in adults 50 years of age and older caused by Streptococcus pneumoniae serotypes 1, 3, 4, 5, 6A, 6B, 7F, 9V, 14, 18C, $19 \mathrm{~A}, 19 \mathrm{~F}$ and $23 \mathrm{~F}$.

Simponi (golimumab by Janssen Inc.) is now approved for reducing signs and symptoms, inhibiting the progression of structural damage and improving physical function in adult patients with moderately to severely active psoriatic arthritis. Simponi can be used in combination with methotrexate in patients who do not respond adequately to methotrexate alone. It is also approved in combination with methotrexate for reducing signs and symptoms and improving physical function in adult patients with moderately to severely active rheumatoid arthritis and inhibiting the progression of structural damage in adult patients with moderately to severely active rheumatoid arthritis who had not previously been treated with methotrexate.

Xarelto (rivaroxaban by Bayer Inc.) is now approved for the treatment of deep vein thrombosis without symptomatic pulmonary embolism.

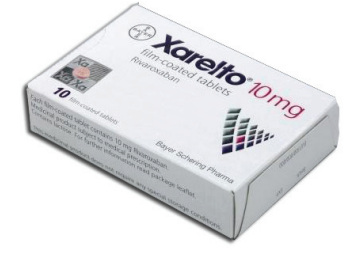

CHANGE IN INDICATION

Rasilez (aliskiren by Novartis Pharmaceuticals Canada Inc.) is no longer approved 
to be used concomitantly with ARBs and ACEIs. Rasilez may be used alone or concomitantly with thiazide diuretics or dihydropyridine calcium channel blockers for the treatment of mild to moderate essential hypertension.

\section{NEW DOSAGE FORM}

Nucynta IR (tapentadol $50 \mathrm{mg}, 75 \mathrm{mg}$ and $100 \mathrm{mg}$ tablets by Janssen Inc.) is a new immediate-release formulation available for the management of moderate to severe acute pain in adults.

\section{NEW GENETICS}

Bisoprolol $5 \mathrm{mg}, 10 \mathrm{mg}$ tablets are now available from Sandoz Canada Inc. This is a new alternative to Monocor.

Cyclobenzaprine $10 \mathrm{mg}$ tablet is now available from Auro Pharma Inc. This is a new alternative to Flexeril.

Entacapone $200 \mathrm{mg}$ tablet is now available from Sandoz Canada Incorporated. This is a new alternative to Comtan.

Lamivudine $150 \mathrm{mg}, 300 \mathrm{mg}$ tablets are now available from Apotex Inc. This is a new alternative to Epivir.

Latanoprost $50 \mathrm{mcg} / \mathrm{mL}$ ophthalmic solution is now available from GenMed, a division of Pfizer Canada Inc. This is a new alternative to Xalatan.

Mirtazapine $15 \mathrm{mg}, 30 \mathrm{mg}, 45 \mathrm{mg}$ orally disintegrating tablets are now available from Auro Pharma Inc. This is a new alternative to Remeron RD.

Nabilone $0.25 \mathrm{mg}, 0.5 \mathrm{mg}$ and $1 \mathrm{mg}$ capsules are now available from Pharmascience Inc. and Ranbaxy Pharmaceuticals Canada Inc. This is a new alternative to Cesamet.

Nevirapine $200 \mathrm{mg}$ tablet is now available from Auro Pharma Inc. This is a new alternative to Viramune.
Risedronate $150 \mathrm{mg}$ tablet is now available from Apotex Inc. This is a new alternative to Actonel.

Rosuvastatin $5 \mathrm{mg}, 10 \mathrm{mg}, 20 \mathrm{mg}, 40$ mg tablets are now available from Cobalt Pharmaceuticals Company. This is a new alternative to Crestor.

Terbinafine $250 \mathrm{mg}$ tablet is now available from Auro Pharma Inc. This is a new alternative to Lamisil.

Venlafaxine XR $37.5 \mathrm{mg}, 75 \mathrm{mg}, 150 \mathrm{mg}$ capsules are now available from GenMed, a division of Pfizer Canada Inc. This is a new alternative to Effexor XR.

\section{DISCONTINUED PRODUCTS}

Benzac AC 5, Benzac W Wash 5, Benzac W5 by Galderma Canada Inc. have been discontinued. Benzac AC 10 and Benzac W Wash 10 continue to be available. Benzoyl peroxide $5 \%$ preparations are available over-the-counter from various brands.

Benzydamine $0.15 \%$ oral solution from Apotex Inc. has been discontinued. The oral solution continues to be available from other generic companies.

Carbamazepine $200 \mathrm{mg}$ tablet by Apotex Inc. has been discontinued. It continues to be available from other generic companies.

Clonidine $0.025 \mathrm{mg}, 0.1 \mathrm{mg}$ and $0.2 \mathrm{mg}$ oral tablets from Apotex Inc. have been discontinued. The tablets continue to be available from other generic companies.

Desocort $0.05 \%$ ointment by Galderma Inc. has been discontinued. Desonide $0.05 \%$ ointment continues to be available from Pharmascience Inc.

Indomethacin $25 \mathrm{mg}$ and $50 \mathrm{mg}$ capsules from Apotex Inc. have been discontinued. The capsules continue to be available from other generic companies.

Metoclopramide $5 \mathrm{mg}$ and $10 \mathrm{mg}$ tablets from Apotex Inc. have been discontinued. The tablets continue to be available from other generic companies.

PremPlus $0.625 \mathrm{mg} / 2.5 \mathrm{mg}$ and 0.625 $\mathrm{mg} / 5 \mathrm{mg}$ from Wyeth LLC have been discontinued. The individual components (conjugated estrogens $0.625 \mathrm{mg}$ and medroxyprogesterone $2.5 \mathrm{mg}$ and $5 \mathrm{mg}$ ) continue to be available individually.

\section{NEW STRENGTHS}

Acuvail (ketorolac tromethamine ophthalmic solution by Allergan Inc.) is a topical nonsteroid anti-inflammatory agent now available in $0.45 \% \mathrm{w} / \mathrm{v}$. Other strengths on the market by Allergan Inc. include $0.4 \%$ $\mathrm{w} / \mathrm{w}$ (Acular LS) and 0.5\% (Acular).

Tamiflu (oseltamivir by Hoffmann-La Roche Limited) is now available in $6 \mathrm{mg} / \mathrm{mL}$ powder for oral suspension. It is also available in $12 \mathrm{mg} / \mathrm{mL}$ powder for oral suspension and in capsules ( $30 \mathrm{mg}, 45 \mathrm{mg}, 75 \mathrm{mg}$ ).

\section{CHANGES IN DOSING}

The starting dose of Revolade (eltrombopag by GlaxoSmithKline Inc.) is $25 \mathrm{mg}$ once daily in ITP patients with liver impairment, specifically Child-Pugh score 5 to 9 . Previously, the Child-Pugh score was 7 to 9. For any dose increase in patients with liver impairment (Child-Pugh score $>5$ ), health care providers are advised to wait a minimum of 3 weeks before increasing the dose. Revolade continues to be contraindicated in patients with Child-Pugh score $\geq 10$.

\section{NEW COMBINATION PRODUCTS}

Tactuo is a topical gel that is a combination of adapalene $0.1 \% \mathrm{w} / \mathrm{w}$ and benzoyl peroxide $2.5 \% \mathrm{w} / \mathrm{w}$ by Galderma Canada Inc.

Alka Bhalla is a pharmacist consultant with the NIHB in Ottawa and the West Carleton Family Health Team in Carp, ON. 